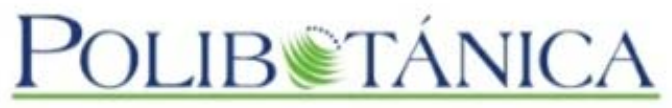

\title{
Polibotánica
}

ISSN electrónico: 2395-9525

polibotanica@gmail.com

Instituto Politécnico Nacional

México

http://www.polibotanica.mx

\section{ARVENSES EN UN SUELO CULTIVADO CON CAÑA DE AZÚCAR CON FERTILIZACIÓN MINERAL Y ABONO VERDE}

\section{WEEDS IN A SOIL CULTIVATED WITH SUGAR CANE WITH MINERAL FERTILIZATION AND GREEN MANURE}

S. Naranjo Landero, J. J. Obrador Olán, E. García López, A. Valdez Balero y V. I. Domínguez Rodríguez ARVENSES EN UN SUELO CULTIVADO CON CAÑA DE AZÚCAR CON FERTILIZACIÓN MINERAL Y ABONO VERDE

WEEDS IN A SOIL CULTIVATED WITH SUGAR CANE WITH MINERAL FERTILIZATION AND GREEN MANURE

POLIBETANICA Instituto Politécnico Nacional
Núm. 50: 119-135 México. Agosto 2020

DOI: $10.18387 /$ polibotanica.50.9

cC (i) (2) Este es un artículo de acceso abierto bajo la licencia Creative Commons 4.0 Atribución-No Comercial (CC BY-NC 4.0 Internacional). 


\section{ARVENSES EN UN SUELO CULTIVADO CON CAÑA DE AZÚCAR CON FERTILIZACIÓN MINERAL Y ABONO VERDE}

\section{WEEDS IN A SOIL CULTIVATED WITH SUGAR CANE WITH MINERAL FERTILIZATION AND GREEN MANURE}

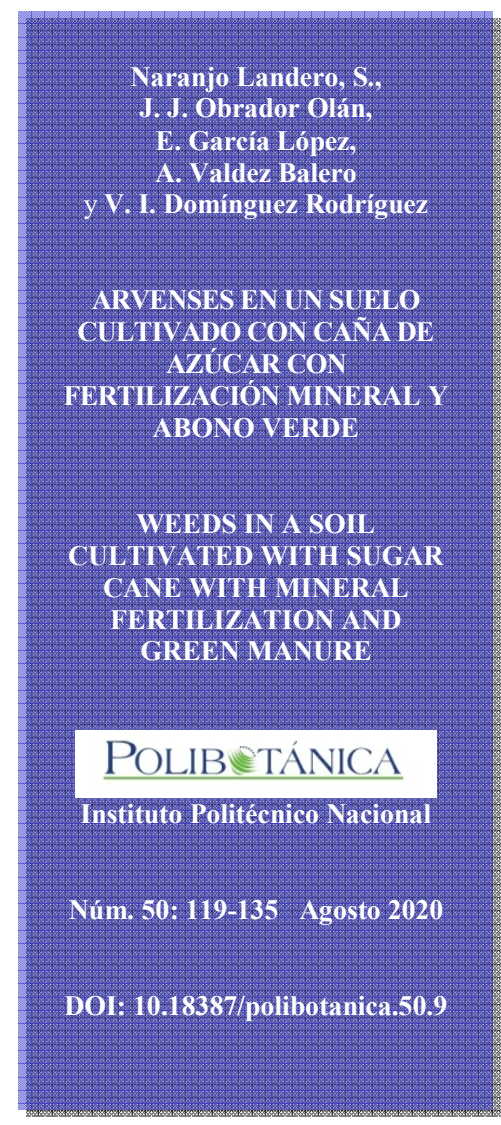

\author{
S. Naranjo Landero \\ Programa de Maestría en Ciencias en Producción Agroalimentaria en el Trópico \\ (PROPAT), Colegio de Postgraduados, Campus Tabasco. \\ J. J. Obrador Olán \\ E. García López / rogarlopez@colpos.mx \\ A. Valdez Balero \\ Colegio de Postgraduados, Campus Tabasco. \\ Carr. Cárdenas - Huimanguillo, Km. 3.5, Cárdenas, \\ Tabasco, México. CP 86500. \\ V. I. Domínguez Rodríguez \\ Universidad Juárez Autónoma de Tabasco. División Académica de Ciencias \\ Biológicas. Carretera Villahermosa-Cárdenas Km. $0.5 \mathrm{~S} / \mathrm{N}$ \\ Entronque a Bosques de Saloya, 86150 Villahermosa, Tab.
}

RESUMEN: Las arvenses son capaces de producir cambios significativos en la estructura, composición y procesos del agroecosistema, pudiendo afectar el rendimiento del cultivo de caña de azúcar en $20-25 \%$ o hasta $97.5 \%$ si la competencia por nutrientes, agua y luz es permanente. Las labores que se realizan para su eliminación, control manual, mecánico y químico, incrementan los costos de producción. En este sentido, la incorporación de abonos verdes resulta benéfica al favorecer el control de arvenses, aumentar la materia orgánica del suelo, mejorar sus propiedades físicas, químicas y biológicas, aumentar la disponibilidad de nutrientes y reducir el uso de fertilizantes minerales. El objetivo del presente estudio fue evaluar el comportamiento de la comunidad de arvenses en caña de azúcar, fase semilla, utilizando Sesbania herbacea como abono verde. Se realizó un diseño de bloques completos al azar con tres tratamientos: 1) abono verde de $S$. herbacea (AV) solo; 2) fertilización nitrogenada NPK (16060 80) y 3) fertilización PK (00 60 80) + AV, y cuatro repeticiones, donde se realizaron muestreos de arvenses en cinco fechas, con los datos registrados se calcularon índices de diversidad: riqueza (S), diversidad $\left(\mathrm{H}^{\prime}\right)$, Uniformidad (E) y de valor de importancia (IVI) de las arvenses. Los valores más altos de $\mathrm{S}$ se observaron en el mes de noviembre en los tres tratamientos, pero siempre fueron mayores en el tratamiento $2(\mathrm{NPK}) ; \mathrm{AV}$ y $\mathrm{PK}+\mathrm{AV}$ mostraron los valores más bajos en octubre y diciembre. H' varió de baja a media, en todos los tratamientos los valores más altos se observaron en el último muestreo. E varió de media a alta, resaltando su relación inversa con S. Los valores de los índices de diversidad no mostraron diferencias estadísticas entre tratamientos en el periodo estudiado, por lo que se recomienda hacer un seguimiento a largo plazo. Las especies de mayor importancia en la comunidad fueron C. rotundus, J. tamnifolia, P. niruri e I. triloba.

Palabras clave: Malezas, Saccharum spp, Sesbania herbacea, diversidad, IVI. 
ABSTRACT: Weeds are capable of producing significant changes in the structure, composition and processes of the agroecosystem, being able to affect the yield of the sugarcane crop by 20 $25 \%$ or up to $97.5 \%$ if the competition for nutrients, water and light is permanent. The tasks carried out for its elimination, manual, mechanical and chemical control, increase production costs. In this sense, the incorporation of green manures is beneficial in favoring weed control, increasing the organic matter of the soil, improving its physical, chemical and biological properties, increasing the availability of nutrients and reducing the use of mineral fertilizers. The aim of the present study was to evaluate the behavior of the community of weeds in sugar cane, seed phase, using Sesbania herbacea as green manure. A randomized complete block design was carried out with three treatments: 1) S. herbacea (AV) green manure alone; 2) NPK nitrogen fertilization (16060 80) and 3) PK fertilization (00 60 80) + AV, and four repetitions, where weeds were sampled on five dates, with the registered data diversity indices were calculated: richness (S ), diversity (H'), uniformity (E) and value of importance (IVI) of weeds. The highest $\mathrm{S}$ values were observed in the month of November in the three treatments, but they were always higher in treatment $2(\mathrm{NPK}) ; \mathrm{AV}$ and $\mathrm{PK}+\mathrm{AV}$ showed the lowest values in October and December. H' varied from low to medium, in all treatments the highest values were observed in the last sampling. E varied from medium to high, highlighting its inverse relationship with $\mathrm{S}$. The values of the diversity indices did not show statistical differences between treatments in the period studied, so it is recommended to do a long-term follow-up. The most important species in the community were C. rotundus, J. tamnifolia, P. niruri and I. triloba.

Key words: Weeds, Saccharum spp, Sesbania herbacea, diversity, IVI.

\section{INTRODUCCIÓN}

La agroindustria de la caña de azúcar en México representa a un importante sector productivo, pues de ella dependen alrededor de tres millones de personas que realizan diversas actividades que van desde la siembra, manejo agronómico, cosecha, industrialización, transporte y comercialización (Velasco-Velasco et al., 2017). Bajo la ley de Desarrollo Rural Sustentable el cultivo es considerado un producto básico y estratégico (CONADESUCA, 2016a).

La caña de azúcar en Tabasco se industrializa en dos ingenios ubicados en la región de la Chontalpa (Presidente Benito Juárez y Santa Rosalía) que se ubican en el municipio de Cárdenas, Tabasco (CONADESUCA, 2019), los rendimientos en campo son bajos en relación a la media nacional debido a que se trata de plantaciones con baja densidad y vulnerables a enfermedades, suelos de baja fertilidad por quemas recurrentes, dosis inadecuadas de fertilizantes, control poco efectivo de malezas, alto costo de insumos, manejo inapropiado del drenaje y casi nulo del riego (Figueroa et al., 2017).

Las propiedades físicas, químicas y biológicas del suelo definen su fertilidad y, aunado al manejo agronómico, los rendimientos de la caña de azúcar (Guerrero-Peña et al., 2017). Los nutrientes absorbidos en mayor cantidad durante el crecimiento y desarrollo de las plantas son el nitrógeno $(\mathrm{N})$, fósforo $(\mathrm{P})$ y potasio $(\mathrm{K})$, éstos deben agregarse en cantidades óptimas para mantener la fertilidad química del suelo y el buen rendimiento del cultivo. Sin embargo, en la búsqueda de mejores rendimientos, el uso de fertilizantes en la agricultura ha ocasionado efectos nocivos al ambiente, a la salud y a los cultivos mismos (Yousaf et al., 2017) por lo que se requiere el ajuste de las dosis de fertilización con base en los rendimientos potenciales, al menos de N, P y K (Tukaew et al., 2016).

Otro problema de importancia en los cultivos es la presencia de arvenses (malezas), ya que intervienen en el equilibrio ecológico provocando problemas fitosanitarios, crecimiento lento, retraso de la floración y bajos rendimientos (Ramos et al., 2011) aunque su presencia puede influir en la estabilidad de los agroecosistemas (Perdomo et al., 2004). Las arvenses en general ejercen una alta competencia por nutrientes, agua y luz, lo que puede significar una reducción 
en la producción de la caña, de 20-25\% (CONADESUCA, 2016b), de 33 a $66 \%$ o hasta 97,5\% si la competencia es permanente (Rodríguez et al., 2019). En general, las labores culturales que se realizan para el control de las arvenses, entre las que destacan el control manual, control mecánico y control químico, generan altos costos al agricultor y efectos negativos al ambiente (Carrillo et al., 2008). El control manual-mecánico es menos eficiente que el uso de herbicidas, sin embargo, este último ocasiona efectos nocivos a la fertilidad biológica del suelo, contamina los mantos freáticos y tiende a generar resistencia de las arvenses por su utilización intensiva y continua (Ramos et al., 2011). En el control de arvenses es imperiosa la integración exitosa de plantas y animales que puedan optimizar las funciones y los procesos del agroecosistema, tales como: la regulación de los organismos dañinos, el reciclaje de nutrientes, la producción de biomasa y la formación de materia orgánica. En este contexto, el uso de abonos verdes (AV), la cobertura y rotación de cultivos y manejos diversificados (agrosilvopastoriles) se perfilan como alternativas viables (Zhang et al., 2019).

La incorporación de abonos verdes (AV) se realiza esencialmente para controlar las arvenses, incrementar los contenidos de materia orgánica, mejorar la actividad biológica y las propiedades físicas del suelo, aumentar la disponibilidad de nutrientes y reducir el uso de fertilizantes minerales (Kim et al., 2013; Li et al., 2015). Como AV se utilizan generalmente leguminosas, plantas que tienen la capacidad de proporcionar $\mathrm{N}$ a los suelos, mediante la fijación biológica, lo que permite disminuir o evitar el uso de fertilizantes nitrogenados ( $\mathrm{Li}$ et al., 2015), pero sobre todo, se incrementa la fertilidad física, química y biológica y con ello mejora la salud del suelo (Obrador-Olán et al., 2019). Sesbania herbacea (Mill.) McVaugh, ha sido utilizada en rotaciones con hortalizas o cereales, observándose mayor rendimiento, menor requerimiento de control de malezas y menor susceptibilidad a las plagas que el caupí (Vigna unguiculata) (Sheahan, 2013). Es una planta que crece bien en suelos anegados y puede aportar más de $200 \mathrm{~kg} \mathrm{~N} \mathrm{ha}^{-1}$, sin embargo, no existe investigación suficiente que indique su capacidad de competir con las arvenses (Naranjo-Landero, 2017). Por otra parte, (Díaz y Labrada, 1996) resaltan que el uso histórico de herbicidas en el cultivo de caña ha generado cambios en la composición de la flora asociada y, consecuentemente, una reducción en la diversidad de ésta. El objetivo del presente estudio fue evaluar el comportamiento de la comunidad de arvenses en caña de azúcar, fase semilla, utilizando $S$. herbacea como abono verde.

\section{MATERIAL Y MÉTODOS}

El estudio se llevó a cabo en el Campo Experimental del Colegio de Postgraduados, Campus Tabasco, que se ubica en las coordenadas $18^{\circ} 00^{\prime} \mathrm{N}$ y $93^{\circ} 30^{\prime} \mathrm{W}$, a una altitud de $9 \mathrm{msnm}$, en el $\mathrm{km} 21$ de la carretera federal 180 Cárdenas-Coatzacoalcos municipio de Cárdenas, Tabasco. El clima es tropical (cálido-húmedo) con lluvias en verano, con medias anuales de temperatura, precipitación y evaporación de $26.7^{\circ} \mathrm{C}, 2240 \mathrm{~mm}$ y $1400 \mathrm{~mm}$, respectivamente (Palma-Lopez et al., 2007).

El experimento se llevó a cabo en un suelo cañero cultivado con caña de azúcar en ciclo plantilla, utilizando la variedad COLPOS CTMEX 06-039 con un alto tonelaje y resistente a algunas enfermedades. El suelo se mecanizó realizando un barbecho y dos rastreos cruzados a $40 \mathrm{~cm}$ de profundidad para una siembra adecuada y uniforme. El diseño fue de bloques completos al azar con tres tratamientos: 1) abono verde (AV) solo; 2) fertilización nitrogenada NPK (160 60 80) y 3) fertilización PK (00 60 80) con AV, y cuatro repeticiones. Los bloques midieron $10 \times 10 \mathrm{~m}$ cada uno, con surcos a $1.3 \mathrm{~m}$, la superficie total de la parcela grande fue de $1200 \mathrm{~m}^{2}$.

Para conocer el comportamiento de la comunidad de arvenses asociadas al cultivo de caña de azúcar se analizó la composición florística de la comunidad y los valores de índices de diversidad y de valor de importancia, para ello se realizaron cinco muestreos: 1) antes de la preparación del suelo, 2) a la floración, corte e incorporación de $S$. herbacea, 3) 30 y 4) 60 días 
después (dd) de incorporar la leguminosa al suelo, y 5) a la cosecha de la caña, correspondiendo con las siguientes fechas; 31 de agosto, 8 de octubre, 8 de noviembre, 3 de diciembre de 2018 y 25 de abril de 2019, respectivamente. En cada tratamiento y fecha se tomaron tres muestras con la ayuda de cuadros de metal de $50 \times 50 \mathrm{~cm}$, en los cuales se registraron, para cada especie, el nombre (común y/o científico), número de individuos y porcentaje de cobertura de cada especie (Mostacedo y Fredericksen, 2000). Se colectaron todas las plantas que quedaron dentro de los cuadros y se llevaron al Herbario CSAT donde se verificó su identidad taxonómica, mediante el uso de bibliografía especializada y con el apoyo de un microscopio estereoscopio.

Los datos registrados se sistematizaron en Excel 2007 y sirvieron para calcular los índices de Riqueza de especies (S), Diversidad (H') y Uniformidad (E) (Magurran, 1989), y el índice de valor de importancia (IVI) de las arvenses, sus componentes: Densidad (De), Frecuencia (Fr), Dominancia (Do) y sus valores relativos (rDe, $\mathrm{rFr}$ y rDo, respectivamente) (Gámez et al., 2011; Concenço et al., 2016), mediante las siguientes fórmulas:

$\mathrm{S}=$ Número de especies

$$
\begin{aligned}
& H^{\prime}=-\sum p i \ln p i \\
& E^{\prime}=H^{\prime} / \operatorname{In} S
\end{aligned}
$$

$\mathrm{De}=\mathrm{N}^{\circ}$ de plantas por especie/unidad de área $\left(\mathrm{m}^{2}\right)$.

$r D e=D e / D e t *$

$\mathrm{Fr}=\mathrm{N}^{\circ}$ de ocurrencias de una especie $/ \mathrm{N}^{\circ}$ total de sitios muestreados.

$r F r=F r / F r t$

Do $=$ Cobertura de una especie/Área muestreada

$r D o=D O / D o t *$

$I V I=r D e+r F r+r D o$.

s. .t $=$ total

Finalmente, con la finalidad de analizar el comportamiento de los índices de diversidad (S, H' y E) se aplicó un análisis de varianza (ANOVA) a los valores del último muestreo, tomando como testigo el primer muestreo.

\section{RESULTADOS}

Como resultado de los cinco muestreos realizados se generó una lista total de 38 especies que fueron ubicadas en 34 géneros y 18 familias botánicas, las que se enumeran en el Cuadro 1, indicando el tratamiento y muestreo en que fueron localizadas. Al grupo de las dicotiledóneas, o malezas de hoja ancha, correspondieron 25 especies (73.5\%). Las familias mejor representadas fueron: Poaceae con 5 especies, Convolvulaceae y Euphorbiaceae con 4, Fabaceae, Malvaceae y Cyperaceae con 3, Asteraceae, Lamiaceae, Linderniaceae y Phyllanthaceae con 2, las ocho restantes cuentan con una sola especie. De las plantas localizadas en los cuadros destacan $C$. rotundus (coquillo), J. tamnifolia (algodoncillo), P. niruri (tamarindillo) e I. triloba; la primera se colectó en todas las muestras y tratamientos en las cinco fechas. 


\begin{tabular}{|c|c|c|c|c|c|c|c|c|c|c|c|c|c|c|c|c|c|c|}
\hline & & & in & & & & & & के & in & & in in & & & & & & \\
\hline & in & & & & & zin & & & $z_{n}$ & & & $z_{n}$ & & & & & & z \\
\hline & & $\hat{3}$ & & in & & in & & & & & & in & & & & & & in \\
\hline & & $\xi$ & & & fे & & ร & & & & & & & & & & & $\hat{z}$ \\
\hline 子 & & & z & & z & & & & & & & & z & $z$ & & z & & $z$ \\
\hline z & & & & & 子 & & & & & & & 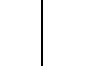 & & & & & & \\
\hline & & & & ले & 이 $\approx$ & & & & & $\hat{m}$ & & ले & & ले & & s & & ले \\
\hline & & & & $\mathrm{m}$ & $z_{m} z_{e}$ & & & $z_{m}$ & & $\mathrm{z}$ & & zol & & 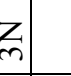 & & & & \\
\hline & & & & 일 & & & હ & & & $\vec{m}$ & & 리 & m & & $\stackrel{m}{m}$ & & 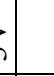 & $\vec{m}$ \\
\hline & & & & & तิ & & & & శ & & & & & & & & & \\
\hline & & $z_{i}$ & $z$ & & $z_{1}$ & $z_{i}$ & $z$ & & & & $z_{N}$ & & & is & & & & \\
\hline & & & & & त & त) & & & 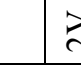 & & & & & & & & & \\
\hline$=$ & & & & $\approx$ & $\Leftrightarrow$ & & & & & & & & $\cong$ & & & & & $\Xi$ \\
\hline$z$ & & & & $z$ & $z$ & & & & & & & & $z$ & & & & & $z$ \\
\hline$\geq$ & & & & $\geq$ & $\geq$ & & & & & & & & $\geq$ & & & & & $2 \geq$ \\
\hline
\end{tabular}

$\theta$

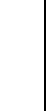

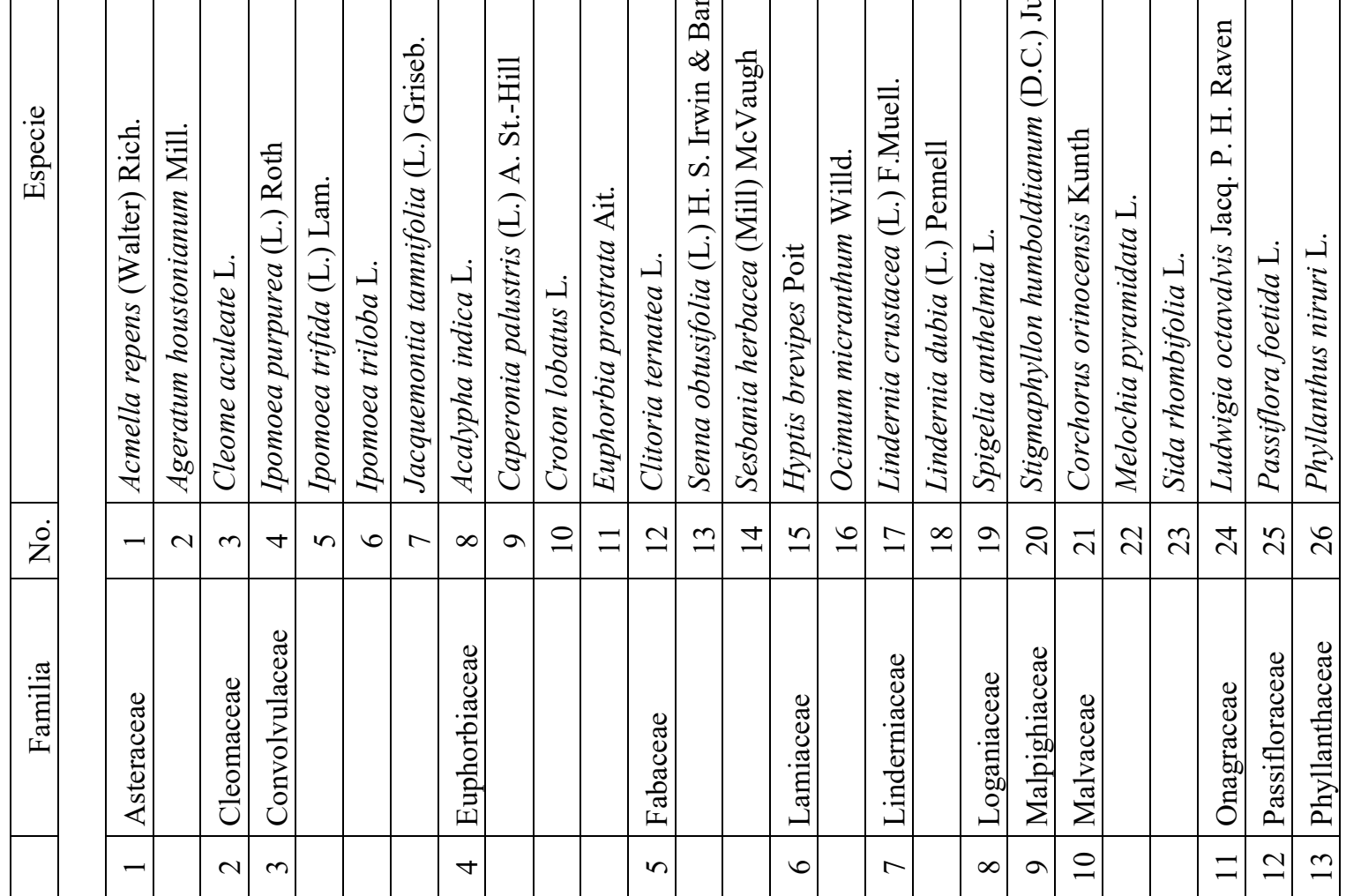




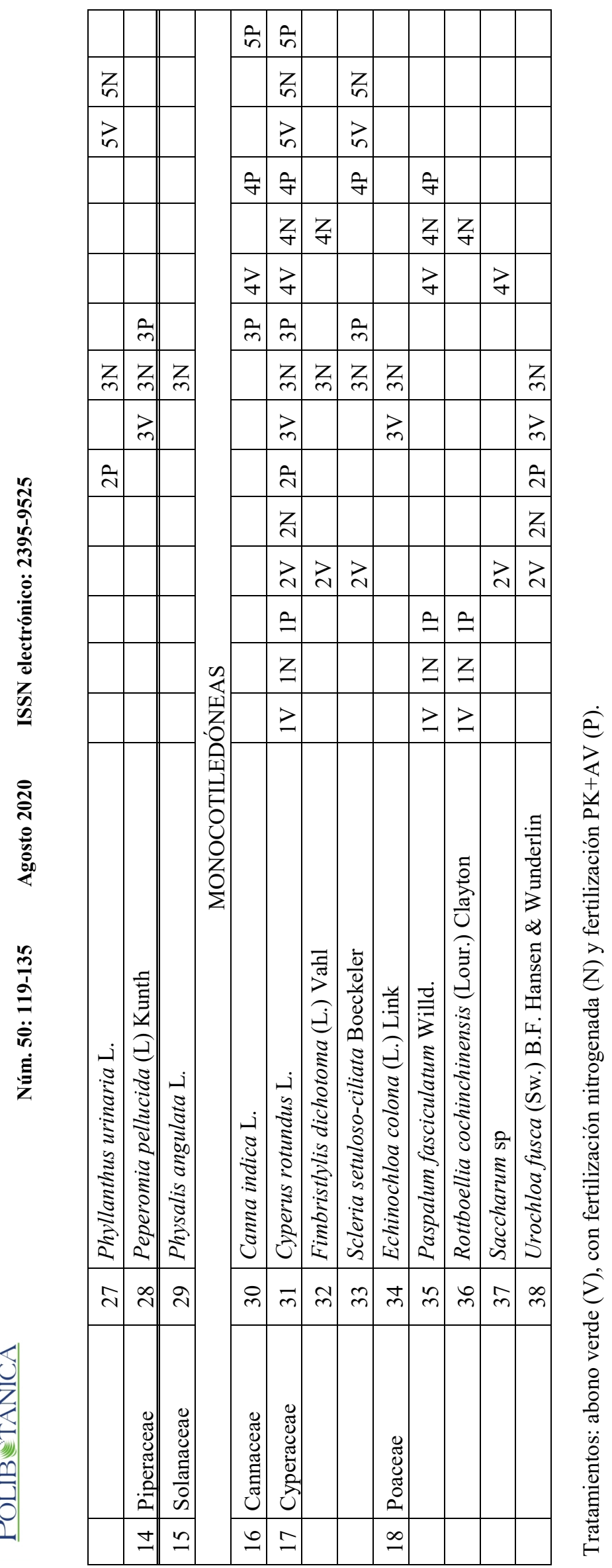


En cuanto a la diversidad de la comunidad de arvenses, en las figuras 1, 2 y 3 se observa el comportamiento de los índices de Riqueza (S), Diversidad (H') y Uniformidad (E). La riqueza, referida al número de especies, presentó los valores más altos en noviembre, 30 dd de la incorporación del AV para los tres tratamientos, pero siempre fue mayor en la parcela con fertilización nitrogenada (NPK) tal vez por la disposición inmediata de $\mathrm{N}$ en el suelo; las parcelas con $\mathrm{AV}$ y $\mathrm{PK}+\mathrm{AV}$ mostraron valores bajos en octubre y diciembre (a la floración del AV y $60 \mathrm{dd}$, respectivamente), probablemente relacionado con la mayor competencia que representó el crecimiento de $S$. herbacea en octubre y por problemas de inundaciones en diciembre.

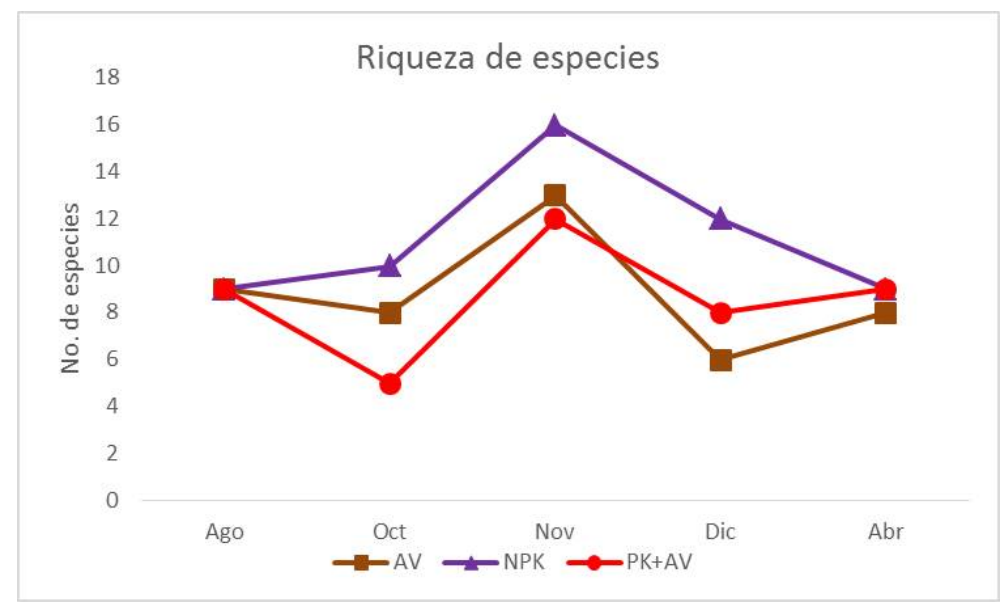

Fig. 1. Comportamiento de la riqueza $(\mathrm{S})$ de especies de arvenses en caña de azúcar con tres tratamientos de fertilización: abono verde (AV), fertilización NPK y fertilización sin N con AV (PK+AV).

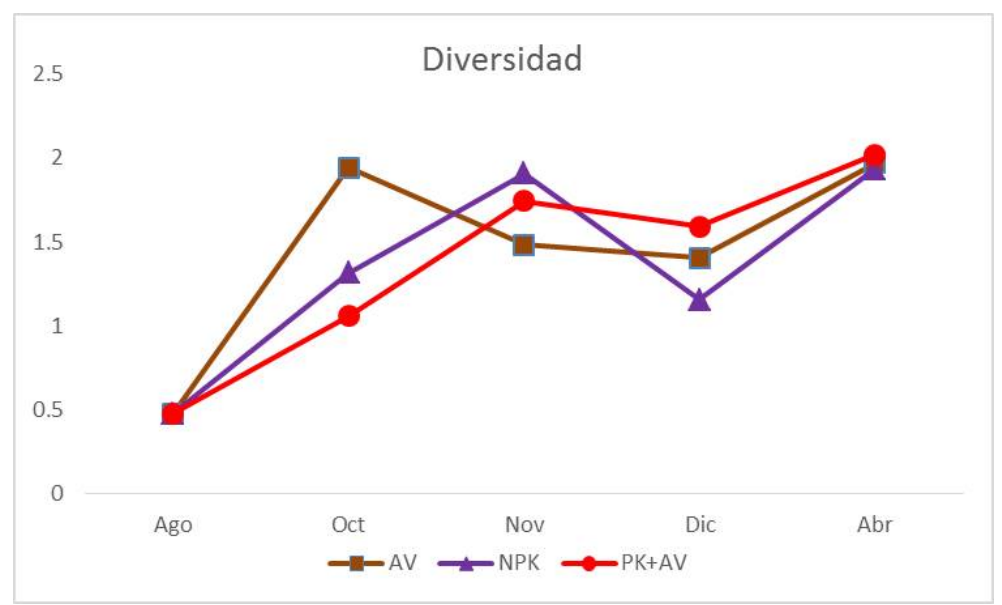

Fig. 2. Comportamiento de la diversidad (H') de especies de arvenses en caña de azúcar con tres tratamientos de fertilización: abono verde (AV), fertilización NPK y fertilización sin N con AV (PK+AV). 


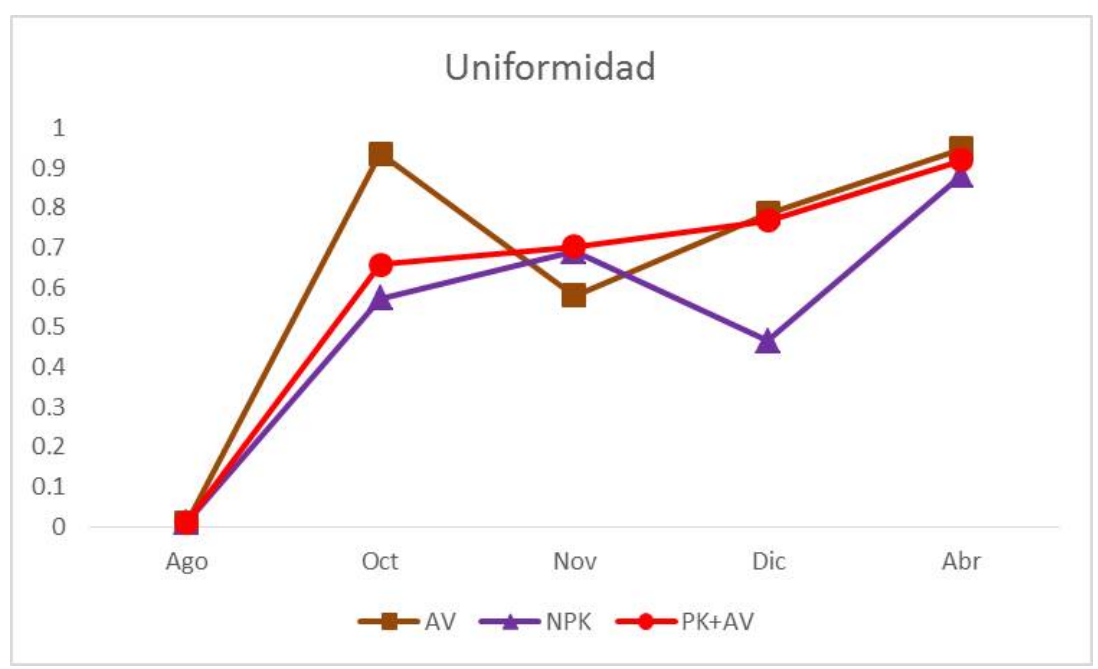

Fig. 3. Comportamiento de la uniformidad (E) de especies de arvenses en caña de azúcar con tres tratamientos de fertilización: abono verde (AV), fertilización NPK y fertilización sin N con AV (PK+AV).

La diversidad (H') de arvenses en las parcelas estudiadas varió de baja en el primer muestreo donde, además de encontrarse pocas especies, se observó dominancia de unas cuantas, a media. El valor más alto se tuvo en octubre en la parcela con AV, coincidiendo con la época de floración y la incorporación de $S$. herbacea. En abril todos los tratamientos presentaron la mayor diversidad, tal vez causada por la disminución en la riqueza de especies (Perdomo et al., 2004).

La Uniformidad (E) de la comunidad de arvenses en las parcelas estudiadas varió de media a alta, resaltando la relación inversa de ésta con la riqueza de especies (Perdomo et al., 2004).

Los análisis de varianza realizados a los valores finales de los índices de diversidad: S, H’ y E no mostraron diferencias estadísticas entre los tratamientos considerados en el presente estudio (figs. 4, 5 y 6 ).

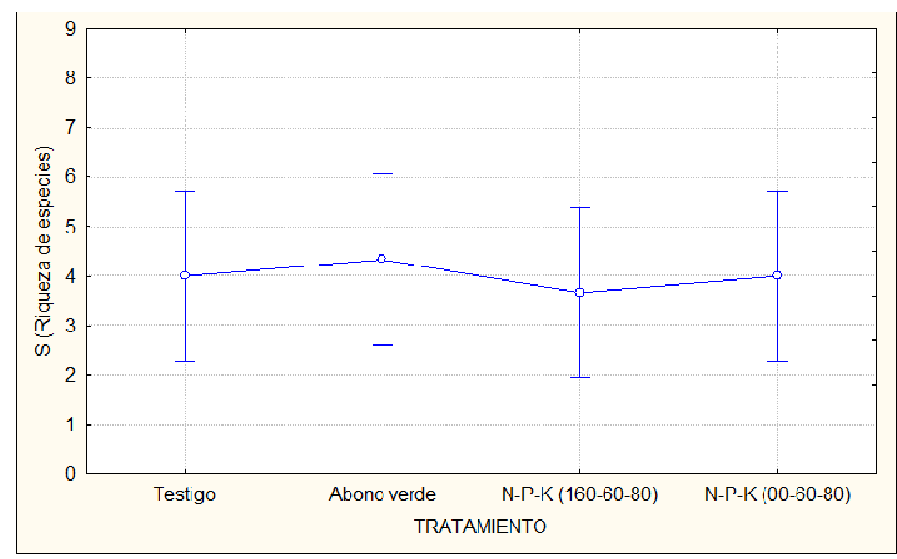

Fig. 4. Análisis de varianza de la riqueza de especies (S) de arvenses en caña de azúcar con tres tratamientos. Las barras verticales indican el intervalo de confianza (0.05). 


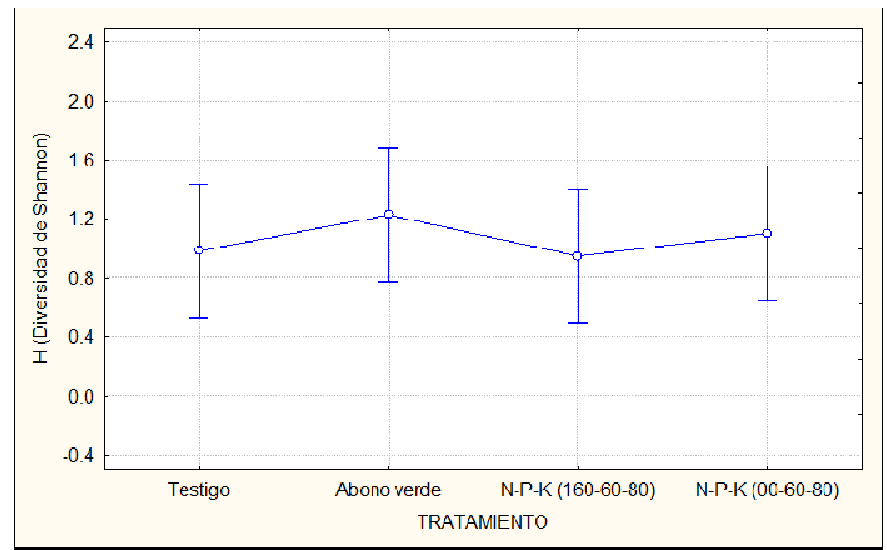

Fig. 5. Análisis de varianza de la Diversidad (H') de arvenses en caña de azúcar con tres tratamientos. Las barras verticales indican el intervalo de confianza (0.05).

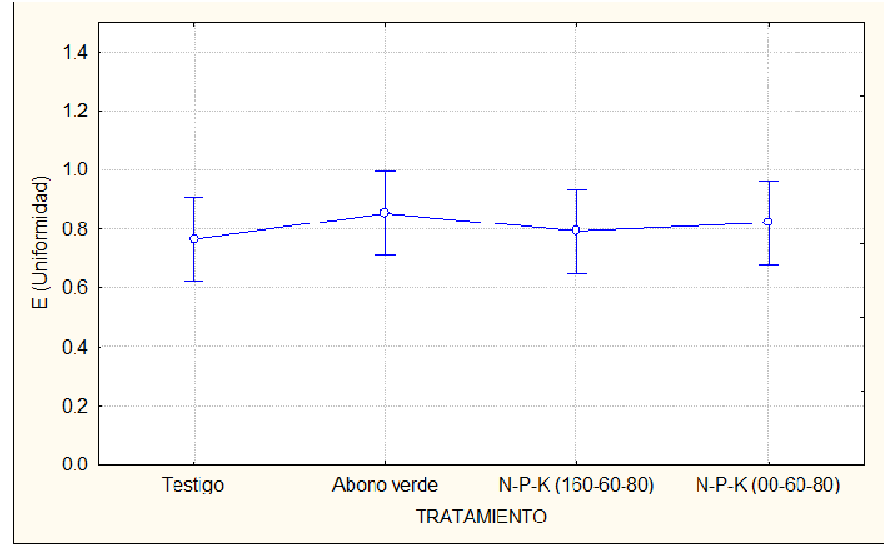

Fig. 6. Análisis de varianza de la Uniformidad (E) de arvenses en caña de azúcar con tres tratamientos. Las barras verticales indican el intervalo de confianza (0.05).

En el cuadro 2 se concentraron los valores del IVI y sus componentes, se consideraron solo las cuatro especies de arvenses más importantes de la comunidad por cada fecha de muestreo y tratamiento. En general, no se detectó que la presencia de alguna(s) especie(s) estuviera influenciada por algún tratamiento. En la primera fecha, cuando aún no se establecían los tratamientos, $P$. fasciculatum tuvo los valores más altos de densidad y frecuencia, pero no de dominancia, donde fue superado por $J$. tamnifolia; para esta fecha destacaron también $C$. rotundus e I. triloba.

Para cuando se realizó el corte de $S$. herbacea, en la parcela AV, J. tamnifolia sigue presentando el valor más alto de dominancia y también de IVI, aunque es superado en frecuencia por U. fasciculata, y en densidad por S. setuloso-ciliata. En donde hubo aplicación de fertilizante nitrogenado (NPK) las dos primeras especies invirtieron su orden, U. fasciculata figura con el mayor IVI, como consecuencia de su alta densidad, J. tamnifolia presentó valores altos de FR y DoR. En la parcela sin nitrógeno C. rotundus fue la más importante, seguida por las dos que ocuparon los primeros lugares en los otros tratamientos. 
Cuadro 2. Índice de valor de importancia (IVI) de las cuatro principales especies de arvenses y valores relativos de sus componentes: Densidad (DeR), Frecuencia (FR) y Dominancia (DoR), en el cultivo de caña con tres tratamientos: $\mathrm{AV}=$ abono verde; 2$) \mathrm{NPK}=$ fertilización nitrogenada y $\mathrm{PK}+\mathrm{AV}=$ fertilización $\mathrm{PK}$ con $\mathrm{AV}$.

\begin{tabular}{|c|c|c|c|c|}
\hline \multicolumn{5}{|c|}{ Muestreo inicial (31/08/2018) } \\
\hline Nombre & DeR & FR & DoR & IVI \\
\hline Paspalum fasciculatum & 47.648 & 26.923 & 37.202 & 111.77 \\
\hline Jacquemontia tamnifolia & 13.228 & 25.071 & 42.544 & 80.843 \\
\hline Cyperus rotundus & 9.389 & 21.795 & 12.36 & 43.543 \\
\hline Ipomoea triloba & 14.286 & 11.111 & 3.509 & 28.906 \\
\hline \multicolumn{5}{|c|}{ Segundo muestreo (8/10/2018) } \\
\hline \multicolumn{5}{|c|}{ Tratamiento 1: AV } \\
\hline Jacquemontia tamnifolia & 19.074 & 29.726 & 33.816 & 82.616 \\
\hline Urochloa fasciculata & 16.296 & 30.159 & 16.311 & 62.766 \\
\hline Scleria setuloso-ciliata & 21.111 & 7.792 & 11.579 & 40.482 \\
\hline Saccharum sp & 14.815 & 5.556 & 12.195 & 32.565 \\
\hline \multicolumn{5}{|c|}{ Tratamiento 2: NPK } \\
\hline Urochloa fasciculata & 41.667 & 17.778 & 23.276 & 82.721 \\
\hline Jacquemontia tamnifolia & 13.79 & 28.611 & 26.542 & 68.942 \\
\hline Cyperus rotundus & 28.571 & 16.667 & 11.494 & 56.732 \\
\hline Ocimum micranthum & 8.333 & 10 & 9.942 & 28.275 \\
\hline \multicolumn{5}{|c|}{ Tratamiento 3: $\mathrm{PK}+\mathrm{AV}$} \\
\hline Cyperus rotundus & 53.495 & 34.815 & 41.303 & 129.61 \\
\hline Urochloa fasciculata & 26.798 & 20.37 & 19.389 & 66.558 \\
\hline Jacquemontia tamnifolia & 9.505 & 20.37 & 24.991 & 54.867 \\
\hline Sesbania herbacea & 8.447 & 21.111 & 12.607 & 42.165 \\
\hline \multicolumn{5}{|c|}{ Tercer muestreo $(8 / 11 / 2018)$} \\
\hline \multicolumn{5}{|c|}{ Tratamiento 1: AV } \\
\hline Cyperus rotundus & 42.435 & 23.875 & 47.232 & 113.54 \\
\hline Lindernia crustacea & 36.597 & 19.272 & 17.919 & 73.788 \\
\hline Ludwigia octavalvis & 5.803 & 11.326 & 5.21 & 22.339 \\
\hline Echinochloa colona & 3.802 & 8.366 & 5.324 & 17.493 \\
\hline \multicolumn{5}{|c|}{ Tratamiento 2: NPK } \\
\hline Cyperus rotundus & 31.862 & 23.219 & 23.79 & 78.872 \\
\hline Lindernia crustacea & 31.535 & 16.952 & 14.321 & 62.808 \\
\hline Jacquemontia tamnifolia & 3.782 & 11.396 & 18.324 & 33.502 \\
\hline Peperomia pellucida & 6.545 & 9.259 & 7.03 & 22.834 \\
\hline \multicolumn{5}{|c|}{ Tratamiento 3: $\mathrm{PK}+\mathrm{AV}$} \\
\hline Cyperus rotundus & 39.201 & 25.943 & 27.151 & 92.294 \\
\hline Jacquemontia tamnifolia & 26.365 & 20.06 & 30.841 & 77.266 \\
\hline
\end{tabular}




\begin{tabular}{|c|c|c|c|c|}
\hline Lindernia crustacea & 9.681 & 11.614 & 7.39 & 28.685 \\
\hline Ludwigia octovalvis & 8.091 & 7.843 & 3.993 & 19.927 \\
\hline \multicolumn{5}{|c|}{ Cuarto muestreo (3/12/2018) } \\
\hline \multicolumn{5}{|c|}{ Tratamiento 1: AV } \\
\hline Cyperus rotundus & 58.143 & 48.611 & 50.618 & 157.37 \\
\hline Saccharum sp & 7.831 & 16.667 & 21.184 & 45.681 \\
\hline Acmella repens & 13.721 & 11.111 & 8.604 & 33.436 \\
\hline Paspalum fasciculatum & 13.375 & 9.722 & 7.93 & 31.028 \\
\hline \multicolumn{5}{|c|}{ Tratamiento 2: NPK } \\
\hline Paspalum fasciculatum & 45.955 & 25.556 & 34.347 & 105.86 \\
\hline Jacquemontia tamnifolia & 20.238 & 24.575 & 42.273 & 87.086 \\
\hline Cyperus rotundus & 8.807 & 21.111 & 11.411 & 41.329 \\
\hline Ipomoea trifida & 4.762 & 8.824 & 3.125 & 16.71 \\
\hline \multicolumn{5}{|c|}{ Tratamiento 3: $\mathrm{PK}+\mathrm{AV}$} \\
\hline Cyperus rotundus & 42.319 & 30.916 & 28.232 & 101.47 \\
\hline Scleria setuloso-ciliata & 19.13 & 21.392 & 14.169 & 54.692 \\
\hline Paspalum fasciculatum & 18.841 & 5.556 & 13.675 & 38.071 \\
\hline Jacquemontia tamnifolia & 6.57 & 13.348 & 13.134 & 33.051 \\
\hline \multicolumn{5}{|c|}{ Quinto muestreo (25/04/2019) } \\
\hline \multicolumn{5}{|c|}{ Tratamiento 1: AV } \\
\hline Ipomoea triloba & 13.095 & 16.667 & 18.403 & 48.165 \\
\hline Caperonia palustris & 15.659 & 16.204 & 11.837 & 43.7 \\
\hline Ipomoea purpurea & 10.897 & 12.037 & 18.571 & 41.505 \\
\hline Phyllanthus niruri & 14.286 & 12.5 & 13.889 & 40.675 \\
\hline \multicolumn{5}{|c|}{ Tratamiento 2: NPK } \\
\hline Lindernia crustacea & 33.81 & 31.111 & 25.789 & 90.71 \\
\hline Phyllanthus niruri & 12.857 & 18.889 & 22.09 & 53.836 \\
\hline Cyperus rotundus & 19.444 & 14.286 & 14.394 & 48.124 \\
\hline Caperonia palustris & 8.333 & 9.524 & 10.606 & 28.463 \\
\hline \multicolumn{5}{|c|}{ Tratamiento 3: $\mathrm{PK}+\mathrm{AV}$} \\
\hline Lindernia crustacea & 22.756 & 18.651 & 19.858 & 61.266 \\
\hline Senna obtusifolia & 16.667 & 16.667 & 20.565 & 53.898 \\
\hline Hyptis brevipes & 16.667 & 14.286 & 14.216 & 45.168 \\
\hline Ipomoea trifida & 12.5 & 14.286 & 14.706 & 41.492 \\
\hline
\end{tabular}

A un mes de la incorporación de la leguminosa al suelo $C$. rotundus presentó el mayor IVI en los tres tratamientos, J. tamnifolia no se encontró en la parcela AV, pero tuvo valores altos de dominancia en las otras dos, donde estuvo en tercer y segundo lugar, respectivamente; en este muestreo también destacaron L. crustacea, con valores altos de DeR y FR y L. octovalvis. 
Al segundo mes de la incorporación de la $S$. herbacea, C. rotundus continuó teniendo los valores más altos en todos los parámetros en los tratamientos AV y fertilización sin $\mathrm{N}$, en el tratamiento NPK fue desplazada al tercer sitio por P. fasciculatum, que resaltó también en los otros tratamientos, y J. tamnifolia que presentó el valor más alto de DoR. En este caso destacaron A. repens en AV, I. trifida en NPK y S. setuloso-ciliata en PK.

En el último muestreo, realizado justo antes de la cosecha de la caña, la especie más importante en los tratamientos fertilizados fue $L$. crustacea, presentado los valores más altos en todos los parámetros, excepto en DoR en PK, donde fue superada por S. obtusifolia; en AV I. triloba tuvo el mayor IVI pero fue superada en De por $C$. palustris, que también figuró en NPK; $P$. niruri tuvo el segundo IVI en NPK y el cuarto en AV donde, a pesar de ser superado por I. purpurea fue más importante en DeR y FR.

\section{DISCUSIÓN}

De las 38 especies que componen la lista florística, 25 corresponden al grupo de las dicotiledóneas, resultados que coinciden con los reportados por Obrador-Olán et al., (2019), (García, 2015) y Pérez et al., (2014) en caña de azúcar. Las tres familias más diversas en esta investigación: Asteraceae, Euphorbiaceae y Poaceae figuran entre las cinco más importantes en los trabajos antes mencionados, en los que Phyllanthaceae aún estaba incluida en Euphorbiaceae. Ramos et al., (2011) menciona que la presencia de integrantes de esas familias está relacionada con las condiciones de clima, suelo y actividades antropogénicas.

Las especies mejor representadas a lo largo de la investigación fueron C. rotundus (coquillo), $J$. tamnifolia (algodoncillo), P. niruri (tamarindillo) e I. triloba. La primera es considerada como una de las peores malezas de mundo, ya que tiene muchas ventajas en la competencia por nutrientes, agua y luz; brota y crece más rápido que muchas plantas cultivables (Doll, 1996), se localiza en más países y regiones del mundo que cualquier otra maleza (Holm et al., 1977) y crece prácticamente en todo tipo de suelo, soportando rangos amplios de $\mathrm{pH}$, humedad y contenido de materia orgánica; su sistema radicular está compuesto de bulbos, rizomas y tubérculos que, además de ocasionar alelopatía, forman cadenas que pueden originar nuevas plantas (Mondragón-Pichardo, 2009). Las cuatro especies suelen prosperar bien en ambientes perturbados y zonas de cultivo y, aunque las tres últimas son de ciclo anual, están bien adaptadas al trópico, donde encuentran las condiciones adecuadas para permanecer una buena parte o todo el año (Vibrans, 2009).

La mayor riqueza de especies en los tratamientos se observó en el mes de noviembre, lo que concuerda con los resultados de Sundara (2011) en caña de azúcar intercalado con caupí (Vigna unguiculata, $V$. radiata y $V$. mungo) donde se observó una reducción significativa de la densidad y peso seco de arvenses durante la etapa temprana de crecimiento de la caña (60 dd siembra). Los mayores valores de $\mathrm{H}^{\prime}$ en todos los tratamientos se observaron en el último muestreo, esto es consecuencia de la disminución en la riqueza de especies, Perdomo et al. (2004), mencionan que, de forma general, se aprecia que cuando los valores de S aumentan la $\mathrm{E}$ se ve disminuida debido a la dominancia de unas cuantas especies. Los cambios en la diversidad de las arvenses suelen estar asociados a labores agrícolas y variación de las condiciones naturales, entre las que destaca que algunas especies necesitan mayor o menor intensidad de luz para su germinación y desarrollo (Ramos et al., 2011).

Los resultados del análisis de varianza (ANOVA) de los valores finales de los índices de diversidad: S, H' y E, no mostraron diferencias estadísticas entre los tratamientos en ninguno de los parámetros estudiados. Al tratarse de caña de azúcar, algunos autores como Díaz y Labrada (1996) resaltan la importancia del uso continuo de herbicidas en el cambio de la composición de especies asociadas al cultivo y una reducción en la diversidad de éstas. En tanto que Pfiffner y Balmer (2011) mencionan que existen varios estudios que muestran el impacto positivo de la 
agricultura ecológica, incluido el uso de AV, sobre la diversidad vegetal y animal de los agroecosistemas; sin embargo, de la Cruz (2015) recomienda hacer un seguimiento a largo plazo de los efectos de los AV, a fin de establecer su incidencia sobre variables biológicas como la reducción de plagas y enfermedades y el cambio en la flora asociada.

Entre las especies más importantes en la comunidad de arvenses asociadas con la caña de azúcar P. fasciculatum, J. tamnifolia e I. triloba son plantas anuales, nativas, que están bien adaptadas y aprovechan las condiciones que el trópico les ofrece para alargar su permanencia en las área de cultivo e interferir en sus rendimientos (Sánchez-Ken, 2012; Vibrans, 2009; Carranza et al., 2008). C. rotundus, en cambio es perenne e introducida, una de las más exitosas e importantes de los trópicos, localizado en más regiones del mundo que ninguna otra maleza (Holm et al., 1977) y, aunque no tolera suelos salinos ni sombra, vive sin dificultad en cultivos de temporal y riego, plantaciones de cítricos, canales, zanjas de drenaje, márgenes de bosques, áreas perturbadas y/o abandonadas (Doll, 1996). Los valores altos de frecuencia y dominancia que J. tamnifolia presentó están relacionados con su capacidad trepadora y rastrera, de modo que uno o pocos individuos pueden ocupar superficies importantes (Carranza, 2008). En cambio, sobre los valores de densidad y frecuencia de L. crustacea y L. octovalvis influyen más las condiciones de humedad del suelo, ya que ambas especies son comunes de sitios anegados (Vibrans, 2009) que, en Tabasco, son característicos de la época de nortes, aunque ObradorOlán et al. (2019) mencionan que L. crustacea sólo ha sido reportada como maleza en cultivo de arroz y malanga, sin embargo, Rojas (2015) reporta que tiene una amplia distribución en regiones tropicales y subtropicales del mundo donde se encuentra en una amplia gama de humedales, siendo capaz de explotar hábitats antropogénicos $\mathrm{y}$, una vez establecida, esta especie tiene el potencial de crecer formando una alfombrilla de hasta $30 \mathrm{~cm}$ de alto.

A. repens y S. setuloso-ciliata fueron importantes en el segundo muestreo en la parcela AV, se trata de malezas comunes de suelos húmedos de pantanos, orillas de arroyos, lagunetas, canales y campos inundados propios de la época en el área de estudio. La última es común en el cultivo de arroz y, además de competir con él, tiene hojas cortantes que dificultan su control manual; pero su biomasa puede servir para mejorar el suelo en sitios húmedos (Rojas, 2010; Rzedowski y Rzedowski 2008). S. obtusifolia aparece en el último muestreo y, aunque se reporta como anual, realmente puede localizarse todo el año en orillas de cultivos y caminos, zanjas, pastizales y terrenos baldíos; Rojas (2010) la reporta con potencial como AV. Caso similar es el de $C$. palustris, se reporta como anual o perenne, Koger et al. (2004) la señalan como muy productiva, ya que cada planta puede producir hasta 900 semillas y al menos $90 \%$ de ellas son viables, su desventaja es presentar dormancia que no se rompe fácilmente; la temperatura óptima para su germinación va de 30 a $40{ }^{\circ} \mathrm{C}$, pero no sucede en condiciones de inundación, aunque las plántulas pueden sobrevivir a ella por varias semanas. Finalmente, I. purpurea ha sido registrada como maleza al menos en 25 cultivos, en los que puede formar poblaciones densas que suelen enredarse en las plantas de cultivo, dificultando su cosecha e incrementando el costo de producción (Villaseñor y Espinosa, 1998).

\section{CONCLUSIONES}

La composición florística del área consistió de 38 especies, 34 géneros y 18 familias botánicas, las mejor representadas fueron Poaceae, Convolvulaceae y Euphorbiaceae. C. rotundus (coquillo), J. tamnifolia (algodoncillo), P. niruri (tamarindillo) e I. triloba fueron las especies mayormente registradas.

Los parámetros de diversidad, S, H' y E, de la comunidad de arvenses no mostraron variaciones importantes en los tratamientos ni en las diferentes fechas. Tampoco hubo diferencia estadística entre tratamientos al finalizar el experimento. 
Respecto al IVI, no se detectó que la presencia de alguna especie estuviera influenciada por los tratamientos, las de mayor importancia fueron plantas nativas de ciclo anual, excepto $C$. odoratus que es introducida y perenne, que tienen periodos largos de permanencia en el área.

\section{AGRADECIMIENTOS}

Al Consejo Nacional de Ciencia y Tecnología (CONACYT) por la beca para la realización de la Maestría en Ciencias de la primera autora.

\section{LITERATURA CITADA}

Carranza, E. (2008). Convolvulaceae II. Flora del bajío y de regiones adyacentes; Fascículo 155. Retrieved from http://inecolbajio.inecol.mx/floradelbajio/documentos/fasciculos/ ordinarios/Convolvulaceae 155.pdf

Carranza, E., Rzedowski, R. J., y Calderón, de R. G. (2008). Diversidad del género Ipomoea L. (Convolvulaceae) en el estado de Michoacán, México. Retrieved from http://bibliotecasibe.ecosur.mx/sibe/book/000047405

Carrillo, 'A. E., Vera, E. J., Alamilla, M. J. C., Obrador, O. J. J., y Aceves, N. E. (2008). Cómo aumentar el rendimiento de la caña de azúcar en Campeche - SIBE. Retrieved from http://bibliotecasibe.ecosur.mx/sibe/book/000046816

CONADESUCA. (2019). 6to. Informe Estadístico del Sector Agroindustrial de la Caña de Azúcar en México Zafras 2009-2010 / 2018-2019. https://doi.org/10.1017/CBO9781107415324.004

CONADESUCA (Comité Nacional para el Desarrollo Sustentable de la Caña de Azúcar). (2016a). Atlas de la agroindustria de la caña de azúcar 2015. Retrieved from http://www.conadesuca.gob.mx/atlas/Atlas_HD.pdf

CONADESUCA (Comité Nacional para el Desarrollo Sustentable de la Caña de Azúcar). (2016b). Identificación de paquetes tecnologicos para el cultivo de caña de azucar en las regiones cañeras de México.

Concenço, G., Leme Filho, J. R. A., Silva, C. J., Marques, R. F., Silva, L. B. X., \& Correia, I. V. T. (2016). Ocorrência de plantas daninhas em cana de açúcar em função de variedade e manejo do palhiço. Planta Daninha, 34(2), 219-228. https://doi.org/10.1590/S0100-83582016340200003

de la Cruz, C. (2015). Contribución de abonos verdes a la producción sostenible de caña de azúcar Saccharum officinarum (L.), en la hacienda La Floresta del municipio de Bugalagrande -Valle del Cauca. Universidad Nacional Abierta y a Distancia. Escuela de Ciencias Agrícolas, Pecuarias y Del Medio Ambiente, (04), 1-61.

Díaz, J. C., \& Labrada, R. (1996). Retrieved from Manejo de Malezas en Cultivos Industriales website: http://www.fao.org/3/t1147s00.htm\#Contents

Doll, D. J. (1977). Capítulo 4. Gramíneas y ciperáceas; Cyperus rotundus L. Retrieved November 6, 2019, from http://www.fao.org/3/T1147S/t1147s08.htm\#referencias4_2

Figueroa, R. K. A., García, G. A. M. T., Mayett, M. Y., Hernández, R. F., y Figueroa, S. B. (2017). Factores que explican el rendimiento de caña de azúcar a nivel municipal en México. Revista Mexicana de Ciencias Agrícolas, 6(6), 1345. https://doi.org/10.29312/remexca.v6i6.581

Gámez, L. A. J., Hernández, M., Díaz, R., y Vargas, J. (2011). Caracterización de la flora arvense asociada a un cultivo de maíz bajo riego para producción de jojotos. Agronomía Tropical, 61(2), 133-139.

García Jiménez P. (2015). Flora asociada al cultivo de caña de azúcar (Saccharum sp.) en el municipio de Cárdenas, Tabasco. Instituto Tecnológico de la Zona Olmeca, Villahermosa, Tabasco, México.

Guerrero-Peña, A., de la Cruz-Pons, A., y Velasco-Velasco, J. (2017, October). Interpretación del análisis de suelos cañeros basado en las relaciones entre propiedades y elementos." 
Agroproductividad, 10(11), 87-92.

Holm, L. G., Plucknett, D. L., Pancho, J. V., y Herberger, J. P. (1977). The world's worst weeds. Distribution and biology. 610.

Kim, S. Y., Gwon, H. S., Park, Y. G., Hwang, H. Y., y Kim, P. J. (2013). Importance of harvesting time of winter cover crop rye as green manure on controlling $\mathrm{CH} 4$ production in paddy soil condition. Functions of Natural Organic Matter in Changing Environment, Vol. 9789400756, pp. 485-488. https://doi.org/10.1007/978-94-0075634-2_88

Koger, C. H., Reddy, K. N., y Poston, D. H. (2004). Factors affecting seed germination, seedling emergence, and survival of texasweed (Crotala palustris). Weed Science, 52(6), 989-995. https://doi.org/10.1614/ws-03-139r2

Li, F., Wang, Z., Dai, J., Li, Q., Wang, X., Xue, C., ... He, G. (2015). Fate of nitrogen from green manure, straw, and fertilizer applied to wheat under different summer fallow management strategies in dryland. 769-780. https://doi.org/10.1007/s00374-015-1023-2

Magurran, A. E. (1989, August). Diversidad ecológica y su medición. Barcelona : Vedra, 1989, 6.

Mondragón-Pichardo, J. (2009). Cyperaceae (Cyperus rotundus L). Retrieved from http://www.conabio.gob.mx/malezasdemexico/cyperaceae/cyperus-rotundus/fichas/ficha.htm

Mostacedo, B., \& Fredericksen, T. S. (n.d.). Manual de Métodos Básico de Muestreo y Análisis en Ecología Vegetal.

Naranjo-Landero, S. (2017). Biomasa aérea y radical de guajillo (Aeschynomene ciliata V.) en un suelo cambisol éutrico de la Chontalpa, Tabasco. Colegio de Postgraduados, Campus Tabasco; InstitutoTecnológico Superior de Comalcalco.

Obrador-Olán, J. J., García-López, E., Almeyda-Santos, L. E., Castelán-Estrada, M., y Carrilloávila, E. (2019). Weeds in a sugar cane soil cultivated with Crotalaria juncea. Planta Daninha, 37, 1-10. https://doi.org/10.1590/s0100-83582019370100002

Palma-Lopez, D. J., Cisnero, D. J., Moreno, C. E., y Rincon-Ramirez, J. A. (2007). Suelos de Tabasco: Su Uso y Manejo Sustentable (PRIMERA ED; C. de P.-I. FUPROTAB, Ed.). Villahermosa, Tabasco. México.

Perdomo, F., Vibrans, H., Romero, A., Domínguez, A., y Medina, J. (2004). Análisis de Sbiodiverhe, una herramienta para estudiar la diversidad de maleza. Rev. Fitotec. Mex 27(1):57-61.

Pérez, D., Ascencio, J., Lazo, J. V., y Castro, M. (2014). Inventario florístico y distribución de malezas presentes en asociación con caña de azúcar antes del cierre del dosel del cultivo en Chivacoa, Estado Yaracuy. Ernstia, 24(1), 25-40.

Pfiffner, L., y Balmer, O. (2011). Organic Agriculture and Biodiversity. In Nutrition \& Food Science (Research I, Vol. 41, pp. 51-58). https://doi.org/10.1108/nfs.2011.01741 faa.014

Ramos, E., Sol, A., Guerrero, A., Obrador, J., y Carrillo, E. (2011). Efecto de Arachis pintoi Sobre las arvenses asociadaal platano macho (Musa AAB), Cárdenas, Tabasco, México. Agronomía Mesoamericana, 22(1), 51-62.

Rodríguez, T. D., Barbosa, G. R. N., y Rodríguez, V. E. (2019). Manejo de arvenses en caña de azúcar, impacto ambiental, efectividad económica y de control. Centro Agrícola, 46(2), 64-71.

Rojas, C. S. (2010). Scleria setuloso-ciliata - ficha informativa. Retrieved November 8, 2019, from http://www.conabio.gob.mx/malezasdemexico/cyperaceae/scleria-setuloso-ciliata/ fichas/ficha.htm

Rojas, S. (2015). Compendio de especies invasoras Lindernia crustacea (pimpinela falsa de Malasia). Retrieved from https://www.cabi.org/isc/datasheet/30864

Rzedowski, J., y Rzedowski, C. G. (2008). Compositae tribu Heliantheae I (géneros AcmellaJefea). Retrieved from http://inecolbajio.inecol.mx/floradelbajio/documentos/ fasciculos/ordinarios/Compositae 157(1).pdf

Sánchez-Ken, G. J. (2012). A synopsis of Digitaria (Paniceae, Panicoideae, Poaceae) in Mexico, including the new species Digitaria michoacanensis. Acta Botanica Mexicana, 101, 127-149. Retrieved from http://sciweb.nybg.org/science2/IndexHerbariorum.asp

Sheahan, C. M. (2013). Bigpod sesbania. Sesbania herbacea (Mill.) McVaugh. USDA-Natural Resources Conservation Service, Cape May Plant Materials Center. 
Recibido:

19/noviembre/2019

Aceptado:

30/julio/2020
Sundara, B. (2011). Agrotechnologies to Enhance Sugarcane Productivity in India. Sugar Tech, Vol. 13, pp. 281-298. https://doi.org/10.1007/s12355-011-0109-x

Tukaew, S., Datta, A., Shivakoti, G. P., \& Jourdain, D. (2016). Production practices influenced yield and commercial cane sugar level of contract sugarcane farmers in Thailand. Sugar Tech, 18(3), 299-308. https://doi.org/10.1007/s12355-015-0403-0

Velasco-Velasco, A., Gómez-Merino, J., Hernández-Cázares, A. S., Salinas-Ruiz, J., y GuerreroPeña, A. (2017). Residuos orgánicos de la agroindustria azucarera: retos y oportunidades. Agroproductividad, 10(11), 99-104.

Vibrans, H. (2009). Malezas de México (CONABIO).

Villaseñor, J. L., y Espinosa, G. F. J. (1998). Catálogo de malezas de México (Ediciones).

Yousaf, M., Li, J., Lu, J., Ren, T., Cong, R., Fahad, S., y Li, X. (2017). Effects of fertilization on crop production and nutrient-supplying capacity under rice-oilseed rape rotation system. Scientific Reports, 7(1), 1-9. https://doi.org/10.1038/s41598-017-01412-0

Zhang, H., Sun, Y., Li, Y., Sun, G., Yuan, F., Han, M., ... Ran, W. (2019). Composted manure and straw amendments in wheat of a rice-wheat rotation system alter weed richness and abundance. Weed Science, 67(3), 318-326. https://doi.org/10.1017/wsc.2018.90 